\title{
Structural Features in Tunica Media of the Aorta in Lamb
}

\author{
Dalma CSIBI ${ }^{1}$, Adrian Florin GAL ${ }^{2 *}$, Cristian RAȚIU ${ }^{3}$, Viorel MICLĂUŞ ${ }^{2}$ \\ ${ }^{1}$ Department of Anatomy, Faculty of Veterinary Medicine, University of Agricultural Sciences and \\ Veterinary Medicine Cluj-Napoca, Calea Mănăştur 3-5, Cluj-Napoca, 400372, Romania. \\ ${ }^{2}$ Department of Histology, Faculty of Veterinary Medicine, University of Agricultural Sciences and \\ Veterinary Medicine Cluj-Napoca, Calea Mănăştur 3-5, Cluj-Napoca, 400372, Romania. \\ ${ }^{3}$ Department of Dentistry, Faculty of Medicine and Pharmacy, University of Oradea, Romania. \\ *corresponding author: adrian.gal@usamvcluj.ro \\ Bulletin UASVM Veterinary Medicine 74(1) / 2017, \\ Print ISSN 1843-5270; Electronic ISSN 1843-5378 \\ DOI:10.15835/buasvmcn-vm: 12624
}

\begin{abstract}
In blood vessels situated just after the heart, an irregular blood flow occurs due to some specific structural elements of the tunica media. The current paper describes the histological aspects of some post-cardiac arterial sections in lamb. The tissue samples were collected from five 30 days old male lambs (Țurcană breed). Histological specimens from different regions of the aorta were harvested (i.e., the ascending aorta, aortic arch, thoracic and abdominal regions of the descending aorta). From the specified regions, small pieces (cca. $0.5 \mathrm{~cm}$ ) were fixed in neutral $10 \%$ buffered formalin. The tissues were subsequently embedded in paraffin wax, sectioned at $5 \mu \mathrm{m}$, and stained with Goldner's trichrome and Verhoeff methods. Tissue analysis was performed using an Olympus system for image acquisition and analysis. Histological appearance of the assessed segments of the aorta in lamb is unusual. Major changes occur in tunica media of the aorta. In the ascending aorta, aortic arch and thoracic regions of the aorta, the histological outline is somewhat the same. The internal region of the media possesses the typical lamellar arrangement. Concerning the outer part of tunica media, the smooth muscle has a tendency to form bundles of various sizes. The muscle islands are not present in the media of abdominal region of the aorta, which exhibits the classic pattern of elastic arteries.
\end{abstract}

Keywords: lamb, tunica media, aorta, elastic artery.

\section{INTRODUCTION}

Blood is pumped by the heart in the arterial system as high pressure intermittent jets. In order to attain the appropriate exchanges between blood and tissues, a continuous blood flow and low pressure are required in capillaries. The particular structure of the arterial wall provides the former circumstances in capillaries. In blood vessels situated just after the heart, an irregular blood flow occurs due to some specific structural elements of the tunica media (e.g., the elastic fibers and a lesser involvement of muscle fibers). Such arteries, in whose tunica media elastic fibers prevail, are also called elastic arteries (Miclăuş, 2012; Gal and Miclăuş, 2013).
Wolinsky and Glagov (1967b) were the first ones to report the medial lamella unit (MLU) in the aortic structure and its role in mice, rats, guinea pig, rabbit, cat, monkey, dog, human, sheep and pig. The mammalian aortic tunica media is typically supposed to be composed of MLU, each including an elastic lamella and the neighboring smooth muscle cells and collagen (Wolinsky and Glacov; 1967a; Awal et al., 1999; Orsi et al., 2004; Akkila et al., 2011; Gupta et al., 2011; Nowrozani, 2011). According to some findings, the number of elastic lamellae varies between certain limits even in the same species. Furthermore, some authors claim that a numerical increase in MLU occurs with age (Junqueira et al., 1992). 
In several primates, including man, the number of aortic medial elastin membranes decreases as the distance from the aortic origin increases (Katzberg, 1966; Ahmed, 1968). Benninghoff suggested that in man, the ascending aorta included 40 to 60 "elastic" sheets, and the abdominal aorta had only about 20 layers (Benninghoff, 1927; Wolinsky and Glagov, 1967b). In humans, a number of papers affirm a variation concerning the quantity of elastic lamellae in the tunica media of elastic arteries, e.g. 40-60 elastic lamellae, 30-50 elastic lamellae, 50-60 elastic lamellae, 40-70 elastic lamellae (Junqueira et al., 1992). According to our knowledge, partial data, concerning the structure of aorta, were reported in animals. As regards the muscular component in tunica media of large arteries, most reports say that smooth muscle cells are arranged in between the elastic lamellae (Junqueira et al., 1992).

Further information on histomorphological arrangement of the aortic wall may improve the understanding of aortic function, and can be used in assessing the changes that happen with age and disease (Ogeng'o et al., 2014). The knowledge concerning the histological pattern of tunica media in large arteries (i.e., post-cardiac arterial sections) in mammals is scarce. Most authors reported little information with reference to the histological pattern in various regions of the first arterial segments departing from the heart. Thus, the current paper describes the histological aspects of some post-cardiac arterial sections in lamb.

\section{MATERIALS AND METHODS}

The tissue samples were collected from five 30 days old male lambs (Țurcană breed, a local variety of sheep). Histological specimens from different regions of the aorta were harvested (i.e., the ascending aorta, aortic arch, thoracic and abdominal regions of the descending aorta). From the specified regions, small pieces (cca. $0.5 \mathrm{~cm}$ ) were fixed in neutral $10 \%$ buffered formalin. The tissues were subsequently embedded in paraffin wax, sectioned at $5 \mu \mathrm{m}$, and stained with Goldner's trichrome and Verhoeff methods (the latter, for highlighting the elastic fibers). Tissue analysis was performed using an Olympus system for image acquisition and analysis, an Olympus BX51 microscope, respectively, connected to an Olympus DP-25 digital photo camera and Olympus Cell B software. Five measurements for each parameter (e.g., layer thickness, vascular wall thickness, muscle islands extension in the thickness of tunica media) in each subject were done and the average value and standard deviation were recorded for each vascular region.

\section{RESULTS}

In the ascending aorta in lamb, media is the largest tunic (i.e., 83.46\%), whereas tunica intima shows an obvious subendothelial layer, and tunica adventitia represents about $15.29 \%$ of the wall thickness (Table 1). The latter displayed a loose aspect. Major structural changes occur in tunica media, which has an uneven aspect regarding the distribution of collagen, elastic and muscle fibers. The mentioned structures are differently arranged in the internal and the external halves of tunica media (Fig. 1).

Concerning the muscle, it is poorly represented in the first quarter of tunica media (i.e., its inner part), somewhat better in the second quarter, decreases again in the third quarter, while in the outermost one tends to be more dominant. True muscle bundles (detectable as various islands in specimens stained with Verhoeff method) are visible in the external part of tunica media (up to $36.37 \pm 8.38 \%$ from the tunica media thickness). The spaces between the muscle bundles are filled with connective fibers (e.g., elastic and collagen fibers) and ground substance. The inner quarters of tunica media exhibit the regular aspect known for this type of arteries, i.e. concentrically arranged elastic lamellae (Fig. 2). As observed, major differences from the standard histological description occur in the outer part of tunica media, features that are visible with Verhoeff stain. Elastic lamellae are somewhat polymorphic concerning their thickness and distribution throughout tunica media.

At the level of the aortic arch, the histological pattern is similar to the one described in the ascending aorta (Fig. 3). Similarly, the histological specimens stained with Verhoeff method demonstrate the existence of muscle fibers in the thickness of tunica media. The muscle fibers arranged in bundles are visible in the outermost part of tunica media. Muscle bundles (visible as some muscle islands) seem slightly better represented in the aortic arch in comparison to the ascending aorta, concerning up to $75.23 \pm 13.74 \%$ 


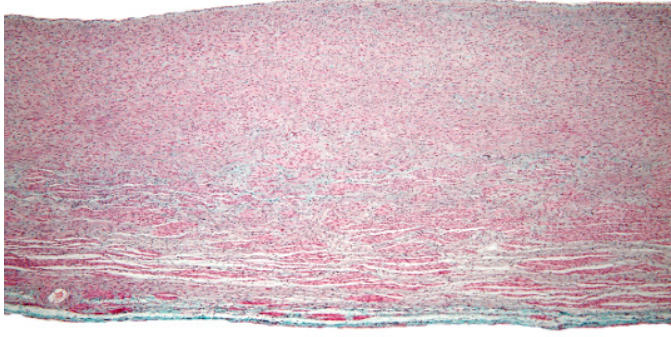

$500 \mu \mathrm{m}$

Fig. 1. Ascending aorta - the proportion between the three layers of the vascular wall (Goldner's trichrome stain).

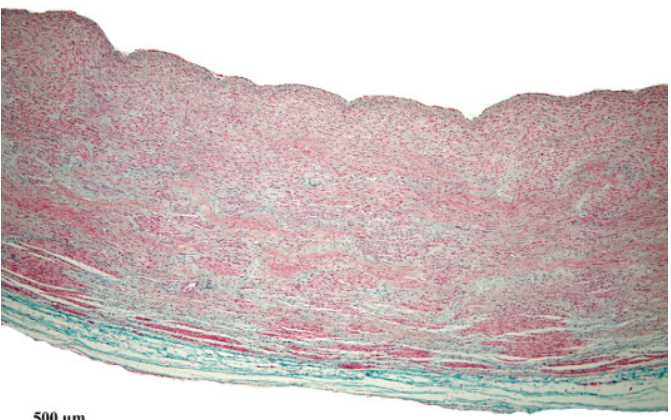

$500 \mu \mathrm{m}$

Fig. 3. Aortic arch - a somehow similar histological pattern to that described in the ascending aorta

(Goldner's trichrome stain).

from the media thickness, in its external part (Fig. 4; Table 1).

In the descending part of the thoracic aorta, the proportion between tunics and their histological display is similar to that of the aortic arch. The uneven aspect of muscle bundles from the outer part of tunica media is present, as in the previous segments (Fig. 5). However, slightly larger muscle bundles are noticed in the external side of tunica media (compared to the same region from previous aortic segments). The insular pattern of the medial smooth muscle occurs in up to $79.53 \pm 13.77 \%$ from the media thickness, mainly into its external part (Fig. 6; Table 1).

The muscle distribution in tunica media of the abdominal descending aorta is completely different in comparison to the previously described aortic segments (Fig. 7). A more uniform histological arrangement is noticed in all quarters

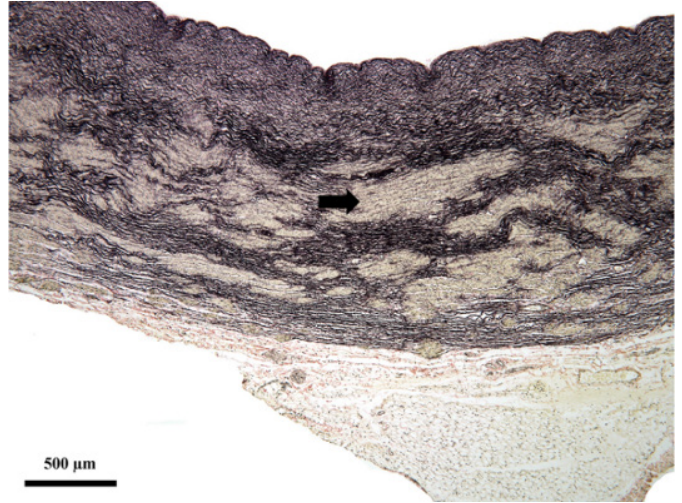

Fig. 2. Ascending aorta - muscle islands in the external part of the tunica media (arrow) and concentrically arranged elastic lamellae into its inner bart (Verhoeff stain).

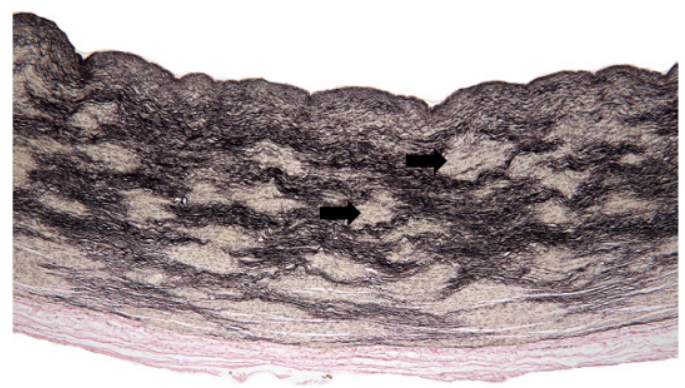

$500 \mu \mathrm{m}$

Fig. 4. Aortic arch - a higher amount of muscle islands (arrows) comparison to the ascending aorta, concerning up to $75 \%$ from the media thickness

(Verhoeff stain).

of tunica media (i.e., in the inner and outer parts of tunica media). In tissue specimens stained with Verhoeff method, a more systematic disposition of the elastic lamellae occurs in all segments of the media. The distance between elastic lamellae is slightly asymmetric, whereas the muscle fibers situated in the inter-lamellar spaces do not have the tendency to form bundles (Fig. 8). Significant differences in comparison to the anterior segments of the aorta are observed in tunica adventitia. Thus, it is considerably thicker than in the previous segments, representing about $24.26 \pm 4.31 \%$ of the wall thickness (in comparison to a variation from 5.39 to $15.29 \%$ in previous aortic regions). Another major change detected here concerns the elastic fibers, which are somehow more numerous in comparison to the previous segments of the aorta (Fig. 8, 9; Table 1). 
Table 1. Diverse measurements in aortic segments in lamb.

\begin{tabular}{|c|c|c|c|c|c|}
\hline Wall layer & Aortic segment & $\begin{array}{l}\text { Layer thickness } \\
\qquad(\mu \mathrm{m})\end{array}$ & $\begin{array}{l}\text { Proportion } \\
\text { comparing to } \\
\text { wall vessel } \\
\text { thickness } \\
(\%) \\
\end{array}$ & $\begin{array}{l}\text { Muscle islands } \\
\text { extension in } \\
\text { the thickness of } \\
\text { tunica media } \\
(\mu \mathrm{m})\end{array}$ & $\begin{array}{c}\text { Proportion of } \\
\text { muscle islands } \\
\text { extension in the } \\
\text { tunica media } \\
(\%) \\
\end{array}$ \\
\hline Intima & $\begin{array}{c}\text { Ascending aorta } \\
\text { Aortic arch } \\
\text { Thoracic descending aorta } \\
\text { Abdominal } \\
\text { descending aorta } \\
\end{array}$ & $\begin{array}{c}18.87 \pm 2.01 \\
14.03 \pm 4.42 \\
6.39 \pm 1.27 \\
6.19 \pm 1.07\end{array}$ & $\begin{array}{l}1.07 \pm 0.10 \\
0.89 \pm 0.28 \\
0.64 \pm 0.15 \\
1.35 \pm 0.26\end{array}$ & $\begin{array}{l}- \\
- \\
-\end{array}$ & $\begin{array}{l}- \\
- \\
-\end{array}$ \\
\hline Media & $\begin{array}{c}\text { Ascending aorta } \\
\text { Aortic arch } \\
\text { Thoracic descending aorta } \\
\text { Abdominal } \\
\text { descending aorta }\end{array}$ & $\begin{array}{c}1466.45 \pm 50.33 \\
1392.09 \pm 55.74 \\
945.03 \pm 83.51 \\
335.63 \pm 27.71\end{array}$ & $\begin{array}{c}83.46 \pm 3.64 \\
88.99 \pm 5.13 \\
94.79 \pm 11.29 \\
73.15 \pm 7.27\end{array}$ & $\begin{array}{c}535.73 \pm 135.00 \\
1042.75 \pm 160.03 \\
744.11 \pm 87.96 \\
-\end{array}$ & $\begin{array}{c}36.37 \pm 8.38 \\
75.23 \pm 13.74 \\
79.53 \pm 13.77 \\
-\end{array}$ \\
\hline Adventitia & $\begin{array}{c}\text { Ascending aorta } \\
\text { Aortic arch } \\
\text { Thoracic descending aorta } \\
\text { Abdominal } \\
\text { descending aorta }\end{array}$ & $\begin{array}{c}268.64 \pm 57.15 \\
189.29 \pm 23.47 \\
54.29 \pm 33.25 \\
111.57 \pm 20.49\end{array}$ & $\begin{array}{c}15.29 \pm 3.33 \\
12.08 \pm 1.43 \\
5.39 \pm 3.04 \\
24.26 \pm 4.31\end{array}$ & $\begin{array}{l}- \\
- \\
- \\
-\end{array}$ & $\begin{array}{l}- \\
- \\
-\end{array}$ \\
\hline
\end{tabular}

\section{DISCUSSION}

Recently, the surgical applications on arteries (e.g., revascularization of the arteries) are extensively used in humans. Accordingly, it is essential to know the structural organization of the aortic tunica media, in order to understand some physicomechanical properties (Avolio et al., 1998; Barry et al., 2007; Farand et al., 2007) and alterations which trigger the aortic pathologies, such as atherosclerosis and aneurysms (Crissman and Pakulski, 1984). A number of animal experimental models were investigated in order to increase the understanding of structural organization of arteries (Ogeng'o et al., 2014; Berry et al., 1974; Kimani and Opole, 1991; Ogeng'o et al., 2009).

Histological appearance of the assessed segments of the aorta (i.e., the ascending aorta, aortic arch, thoracic and abdominal regions of the descending aorta) in lamb is unusual and different from the typical description noticed in most mammals. Major changes occur in tunica media of the aorta. There is an irregular distribution of smooth muscle cells and elastic lamellae in tunica media of the aorta in lamb. Accordingly, significant differences occur between internal and external parts of the media. Thus, in the first three regions of the aorta (i.e., the ascending aorta, aortic arch and thoracic regions of the aorta) the histological pattern in tunica media is relatively the same. The internal region of the media possesses the typical lamellar arrangement that is specific for elastic arteries, whereas the external region is different. Concerning the outer part of tunica media, the smooth muscle has a tendency to form bundles of various sizes with a circular orientation of the myofibers. The muscle bundles are separated by relatively large spaces occupied by connective tissue (i.e., elastic, collagen fibers and associated ground substance). However, a noticeable increase in the muscle bundles occurs in tunica media from the ascending aorta (i.e., $36.37 \pm 8.38 \%$ from the thickness of tunica media) to the aortic arch (i.e., $75.23 \pm 13.74 \%$ from the thickness of tunica media) and thoracic regions of the aorta (i.e., $79.53 \pm 13.77 \%$ from the thickness of tunica media). If in the ascending aorta the muscle (with its insular disposition) exists only in the external part of the media (i.e., concerning approximately $36.37 \%$ from the tunica media thickness), in the thoracic descending aorta and aortic arch the muscle expands on two thirds from the tunica media thickness (i.e., concerning roughly $75.23-79.53 \%$ from the media thickness, into its external part). As a similarity, Ogeng'o et 


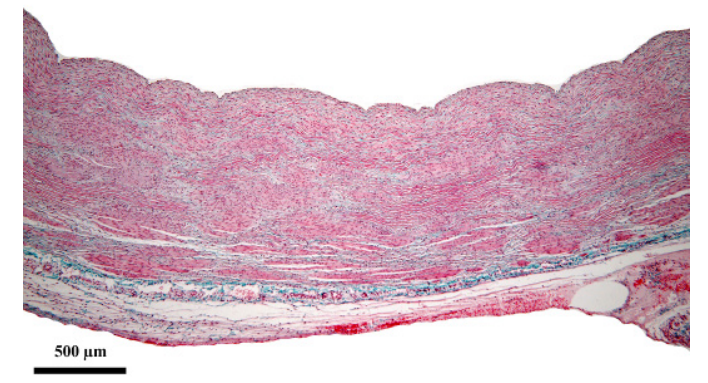

Fig. 5. Descending part of the thoracic aorta the irregular distribution of muscle bundles is still present in the tunica media, resembling the previous segments (Goldner's trichrome).

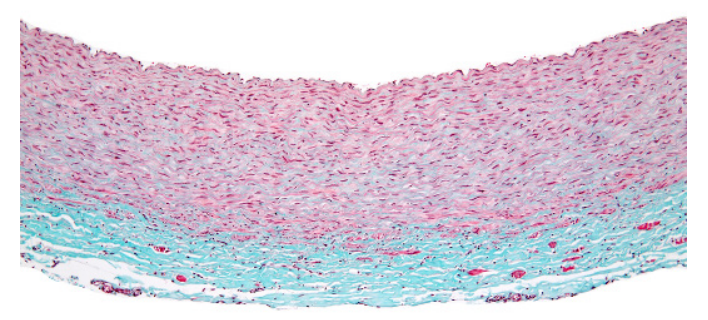

$200 \mu \mathrm{m}$

Fig. 7. Descending part of the abdominal aorta - a totally different pattern can be observed in the tunica media comparing to all previous aortic segments (Goldner's trichrome).

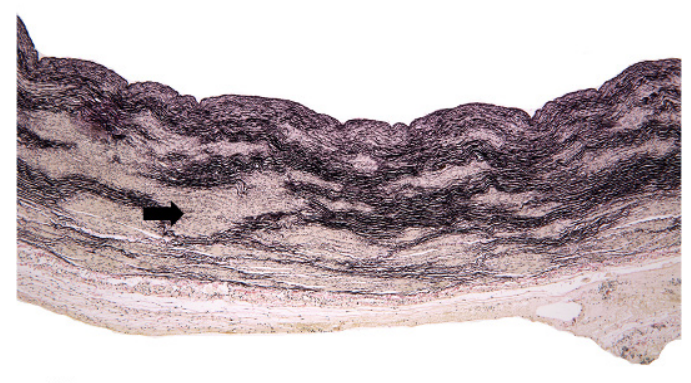

$500 \mu \mathrm{m}$

Fig. 6. Descending part of the thoracic aorta slightly larger muscle islands (arrow) are present in tunica media (Verhoeff stain).

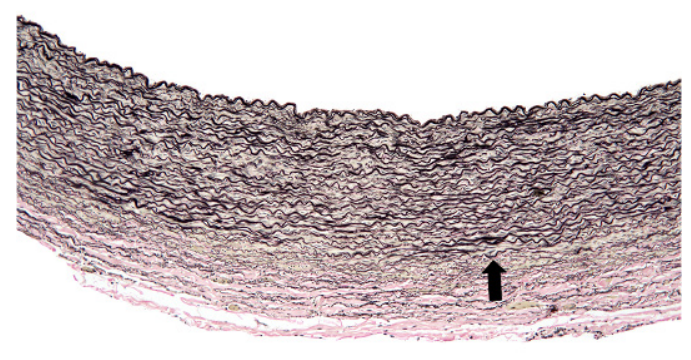

$200 \mu \mathrm{m}$

Fig. 8. Descending part of the abdominal aorta tunica media (arrow suggesting the external limit of media) does not have muscle islands like the former aortic regions; a more regular disposition of the elastic lamellae occurs in the media (Verhoeff stain).

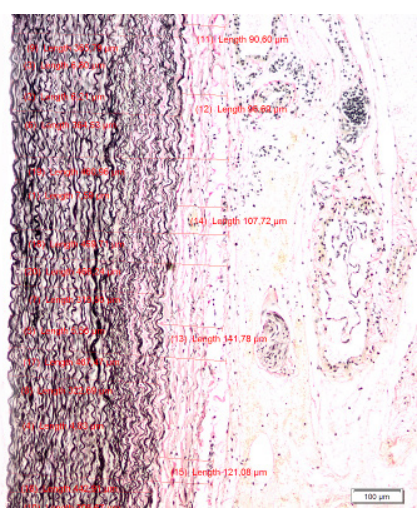

Fig. 9. Descending part of the abdominal aorta the Olympus system for image analysis (Verhoeff stain).

al. (2014) described the aortic tunica media as a heterogeneous mosaic-like structure.

An unexpected structural change is observed in tunica media of the abdominal descending aorta. Thus, the tunica media no longer presents the muscular pattern described in the earlier segments of the aorta. The typical histological arrangement for elastic arteries is observed in tunica media of the suggested region. Elastic circular parallel lamellae are fairly present in all the thickness of tunica media. A limited amount of smooth muscle fibers is present in between 
the elastic lamellae of the tunica media, without the tendency to form bundles. As observed in our description, only the abdominal descending aorta resembles the classical elastic artery described in humans and some other mammals (Wolinsky and Glacov, 1967a; Katzberg, 1966; Ahmed, 1968; Benninghoff, 1927; Wolinsky and Glagov, 1969).

Another variation noted in the inspected regions of the aorta is represented by a progressive increase in thickness of tunica media, from the ascending aorta to the descending thoracic section (i.e., from roughly $83.46 \%$ in ascending aorta to $94.79 \%$ in the thoracic descending aorta; Table 1). Tunica media of the abdominal descending aorta displayed a substantial reduction in thickness (i.e., from approximately $94.79 \%$ in the thoracic descending aorta to roughly $73.15 \%$ into its abdominal region). However, tunica adventitia showed a decline in thickness from the first aortic region to the thoracic descending region (ranging from around $15.29 \%$ in the ascending aorta to about $5.39 \%$ in the thoracic descending aorta). Concerning the descending region of the aorta, an important thickening of the adventitia occurs in comparison to the preceding thoracic region (i.e., from approximately $5.39 \%$ to $24.26 \%$; Table 1 ). Tunica intima did not present major differences from one aortic region to another.

Histological findings of the present report reveal that in the lamb ascending aorta, aortic arch and thoracic descending aorta, tunica media includes an uneven zone in which muscle islands break off elastic lamellae. Our findings are similar to those described in goat, cow and sheep (Ogeng'o et al., 2009; Knieriem, 1967; Fukuda et al., 1984; Malek and Ogeng'o, 2007). However, the present paper further reveals that the muscle islands are no longer present in the abdominal region of the aorta, aspect not described by others.

The specific arrangement of the muscle in the media of the first three segments of the aorta in lamb is enigmatic. The described histological layout of tunica media is not encountered in other mammalian species (with the exception of goat, cow and sheep). The appearance might be linked to a necessity to transform the blood flow from intermittent to continuous. The blood pumping during diastolic period is performed by a passive contraction of the vascular elastic components, which may also be sustained by an active contraction of the muscle. It is possible that this particular situation is related to the existence of forestomachs that do not exist in most species. Ogeng'o et al. (2009) suggest that the smooth muscle islands interlocked with matrix and with adrenergic nerves, and vasa vasora in the proximal regions of the aorta are most likely designed to represent a supplementary pump, for augmenting elastic recoil during diastolic period, a method for strengthening the aortic wall, and to regulate blood flow. Additionally, the former authors state that adrenergic nerves adjust contraction while vasa vasora distribute required oxygen and nutrients. This suggests that the role of the islands implicates active regulated contraction, which may provide a number of functions.

The muscle islands are most likely implicated in regulation of blood flow to the cranial parts of the animal. This presumption is sustained by the idea that in birds, transmural zonation into luminal elastic and adventitial muscular areas allows changes in vessel radius to adjust the effects of severe circulatory changes in the rear part of the body during flight (Berry et al., 1974). An analogous blood flow regulation function has been suggested for the muscular region of the giraffe brachiocephalic and bicarotid trunks (Kimani and Opole, 1991).

\section{CONCLUSION}

The histological appearance of the tunica media of aorta in lamb is unusual in comparison to most mammals. In the ascending aorta, aortic arch and thoracic regions of the aorta, the histological outline is somewhat the same, resembling a mosaic arrangement. Still, the muscle islands are not present in the media of abdominal region of the aorta, which exhibits the classic pattern of elastic arteries. The particular aspect of the media in lamb aorta plays an essential role in support of the dynamism and physicomechanical potency of the vessel. Finally, the histological pattern of the aorta in sheep is somehow different from the one of the aorta in humans. In view of that, the benefits of such a model in the assessment of various human vascular problems are limited.

\section{REFERENCES}

1. Ahmed MM (1968). Microscopic anatomy and the attenuation of elastic tissue in the aortic wall of slow loris (Nycticebus concang concang). Folia Primat 8(3-4):290300 . 
2. Akkila SS, Eleiwe SA, Qassein EG, Abdulaleef HH (2011). Histological changes in the thoracic aorta of adult rats treated with melatonin. Iraqi J Comm Med 24(2):168-173.

3. Avolio A, Jones D, Tafazzolo-Shadpour M (1998). Quantification of alterations in structure and function of elastin in the arterial media. Hypertension 32(1):170175.

4. Awal MA, Prodhan MAA, Asaduzzaman M, Kurohmaru M (1999). Histological studies on the arterial walls of main arteries supplying mammary glands of black Bengal goats (Capra hircus) in Bangladesh. Vet Arhiv 69(6):309-318.

5. Barry M, Touati G, Farag M, Werbrouck A, Foulon P, Caus T, Le Gars D, Laude M, Sevestre H (2007). Biometric and histological study of human, porcine and sheep internal thoracic arteries (reflections on their use in revascularization of arteries of less than four millimeters diameter). Morphologie 91(292):24-28.

6. Benninghoff A (1927). Uber die Beziehungen zwischen elastischen Gerust und glatter Muskulatur in der Arterienwand und ihre funktionelle Bedeutung (in German). Z Zellforsch Mikroskop Anat Abt Histochem 6(3):348-396.

7. Berry CL, Germainand J, Lovell P (1974). Comparison of aortic lamellar unit structure in birds and mammals. Atherosclerosis 19(1):47-59.

8. Crissman RS, Pakulski LA (1984). A rapid digestive technique to expose networks of vascular elastic fibers for SEM observation. Stain Technol 59(3):171-180.

9. Farand P, Garon A, Plante GE (2007). Structure of large arteries: orientation of elastin in rabbit internal elastic lamina, and in elastic lamellae of aortic media. Microvasc Res 73(2):95-99.

10. Fukuda Y, Perraus VJ, Crystal RG (1984). Development of elastic fibers of nuchal ligament, aorta and lung of fetal and postnatal sheep. An ultrastructural and electron microscopic immunohistochemical study. Am J Anat 170(4):597-629.

11. Gal AF, Miclăuş V (2013). Histology. Risoprint Cluj-Napoca, Romania, 165-181.

12. Gupta SD, Gupta SK, Pal DK, Sarawagi R, Gupta P (2011). Microscopic study of aorta in relation of different age groups: an observational study. Int J Biol Med Res 2(1):398-403.
13. Junqueira LC, Carneiro J, Kelly RO (1992). Basic Histology. $7^{\text {th }}$ ed. Connecticut: Appleton \& Lange Norwalk, 216-231.

14. Katzberg AA (1966). The attenuation of elastic tissue in the aorta of the baboon, Papio doguera. Anat Record 154(2):213-219.

15. Kimani JK, Opole IO (1991). The structural organization and adrenergic innervation of the carotid arterial system of the Giraffe (Giraffa Camelopardalis). Anat Rec 230(3):369-377.

16. Knieriem HJ (1967). Electron-microscopic study of bovine atherosclerotic lesions. Am J Pathol 50(6):1035.

17. Malek AKA, Ogeng'o JA (2007). Regional differences in the tunica media of the aorta of the goat (Cabra Ibex). J Anat 210(6):767.

18. Miclăuş V (2012). Histologie specială și embriologie generală (in Romanian). Risoprint Cluj-Napoca, Romania, 7-13.

19. Nowrozani FR (2011). Comparison of abdominal aorta and renal artery in the neonate male dog. J Anim Vet Adv 10(17):2278-2281.

20. Ogeng'o JA, Malek AAK, Kiama SG, Olabu BO (2009). Muscle "islands" in the tunica media of the goat thoracic aorta. Braz J Morphol Sci 26(3-4):171-175.

21. Ogeng'o JA, Malek AK, Ongeti KW, Mwachaka PM (2014). Deviation of the tunica media of aorta of goat from conventional structural concept: Case for including collagen. International Journal of Anatomy and Physiology 3(1):042-047.

22. Orsi AM, Stefanini MA, Crocci AJ, Simoes K, Ribeiro AACM (2004). Some segmental features on the structure of the aortic wall of the dog. Anat Histoly Embryol 33(3):131134.

23. Wolinsky H, Glacov S (1967a). Nature of species differences in the medial distribution of aortic vasa vasorum in mammals. Circ Res 20(4):409-421.

24. Wolinsky H, Glagov S (1967b). Lamellar unit of the aortic medial structure and function in mammals. Circ Res 20(1):99-111.

25. Wolinsky H, Glagov S (1969). Comparison of abdominal and thoracic aortic medial structure in mammals. Circ Res 25(6):677-686. 